\title{
Leaf Volatile Compounds and Associated Gene Expression during Short-Term Nitrogen Deficient Treatments in Cucumis Seedlings
}

\author{
Jie Deng ${ }^{1,+}$, Hong-Jun Yu ${ }^{1,+}$, Yun-Yun Li ${ }^{1}$, Xiao-Meng Zhang ${ }^{1}$, Peng Liu ${ }^{1}$, Qiang Li ${ }^{1, *}$ and \\ Wei-Jie Jiang ${ }^{1,2, *}$ \\ 1 Key Laboratory of Horticultural Crops Genetic Improvement (Ministry of Agriculture), \\ Institute of Vegetables and Flowers, Chinese Academy of Agricultural Sciences, Beijing 100081, China; \\ 115425193@163.com (J.D.); yuhongjun@caas.cn (H.-J.Y.); yijinlidy@163.com (Y.-Y.L.); \\ 13716247546@163.com (X.-M.Z.); pengliucaas@foxmail.com (P.L.) \\ 2 College of Forestry and Horticulture; Xinjiang Agricultural University, Urumqi 830052, China \\ * Correspondence: liqiang05@caas.cn (Q.L.); jiangweijie@caas.cn (W.-J.J.); \\ Tel.: +86-10-8210-8797 (Q.L. \& W.-J.J.) \\ + These authors contributed equally to this work.
}

Academic Editor: Jianhua Zhu

Received: 20 July 2016; Accepted: 8 October 2016; Published: 2 November 2016

\begin{abstract}
Nitrogen $(\mathrm{N})$ is an important macronutrient for plant growth and development, but the regulatory mechanism of volatile compounds in response to $\mathrm{N}$ deficiency is not well understood, especially in cucumber, which consumes excessive $\mathrm{N}$ during growth. In this study, the major volatile compounds from cucumber leaves subjected to $\mathrm{N}$ deficiency were analyzed by GC-MS. A total of 24 volatile components were identified including 15 aldehydes, two ketones, two alkenes, and five other volatile compounds in 9930 leaves. Principal component analysis using volatile compounds from cucumber leaves provided good separation between $\mathrm{N}$-sufficient and $\mathrm{N}$-deficient treatments. The main volatiles in cucumber leaves were found to be $\mathrm{C} 6$ and $\mathrm{C} 9$ aldehydes, especially (E)-2-hexanal and (E,Z)-2,6-nonadienal. (E)-2-hexanal belonged to the $\mathrm{C} 6$ aldehyde and was the most abundant compound, whereas (E,Z)-2,6-nonadienal was the chief component of $C 9$ aldehydes. During $\mathrm{N}$-deficient treatment, short-chain volatile content was significantly improved at 5 day, other volatiles displayed significant reduction or no significantly changes in all sampling points. Improvement of short-chain volatiles was confirmed in the six other inbred lines at 5 day after $\mathrm{N}$-deficient treatments. The expression analysis of 12 cucumber LOX genes and two HPL genes revealed that CsLOX19, CsLOX20, and CsLOX22 had common up-regulated expression patterns in response to $\mathrm{N}$-deficient stress in most inbred lines; meanwhile, most sample points of CsHPL1 also had significant up-regulated expression patterns. This research focused on the relationship between volatiles in cucumber and different nitrogen environments to provide valuable insight into the effect of cultivation and management of the quality of cucumber and contributes to further research on volatile metabolism in cucumber.
\end{abstract}

Keywords: nitrogen deficiency; cucumber; leaf volatile; aldehyde; LOX-HPL pathways

\section{Introduction}

Cucumber (Cucumis satious Linn.) is a widely grown vegetable with a fresh and distinct flavor [1]. During cucumber cultivation, excessive nitrogen $(\mathrm{N})$ fertilizer application is used to achieve a high yield, but it also leads to substantial environmental degradation [2]. $\mathrm{N}$ fertilizer has been the major cost in cucumber production and reduces the income of farmers [3]. Furthermore, excessive application of $\mathrm{N}$ can deteriorate vegetable flavor [4] and decrease plant resistance to pathogens [5,6]. During N-deficient 
treatments, the antioxidant enzyme system (Superoxide dismutase, peroxidase, and catalase) of cucumber seedlings was activated markedly and exhibited higher levels of antioxidants [3,7]. Thus far, researchers have identified 78 volatile compounds from cucumber, including aldehydes, alcohols, esters, alkanes, furfurans, and others [8]. Volatile C6 and C9 aldehydes are considered important factors to the characteristic flavors of green leaves, fruits and vegetables. Plants produce volatile compounds, such as $\mathrm{C} 6$ aldehydes, $\mathrm{C} 9$ aldehydes, and their acetates, through the lipoxygenase (LOX) and hydroperoxidelyase (HPL) pathways [9]. Linoleic and $\alpha$-linolenic acid are the substrates for dioxgenation and subsequent cleavage to obtain $\mathrm{C} 6$ and $\mathrm{C} 9$ volatile aldehydes, which can be further modified by alcohol dehydrogenases (ADH) [10,11]. (E,Z)-2,6-nonadienal (which has a cucumber-like flavor) and (E)-2-hexenal (which has an apple-like flavor) are the main volatile compounds $[8,12]$. Green leaf volatiles (GLVs) have also been reported to play important roles in different biological processes [13-15]. Contents of GLVs display improvement after infection with bacteria and pathogenic fungi [16-18], which indicates that a possible physiological function of these short-chain volatiles is to limit pathogen growth. Moreover, pretreatment with exogenous (E)-2-hexenal resulted in increased resistance against pathogens, most likely as a result of both activation of defense responses and direct inhibition of fungal growth $[17,19]$. In higher plants, short-chain aldehydes are also produced in response to wounding, and they also have important functions during wound healing and pest resistance [20]. Some GLVs were also demonstrated to be signal chemicals, which are produced by environmental stress to induce abiotic-related gene expression as oxidative stress signals [21].

Most prior research has focused on the types of volatile compounds present in cucumber fruit and their potential role in fruit development $[8,22]$. Little information is available about the mechanisms of volatile biosynthesis in cucumber seedlings. In the present study, the effect of nitrogen deficiency on volatile compounds in cucumber leaves was investigated on transcriptional and metabolic level, respectively. These results provide a basis for further studies on the regulation of $\mathrm{C} 6$ and $\mathrm{C} 9$ volatiles in cucumber leaves.

\section{Results and Discussion}

\subsection{Analysis of Cucumber Leaf Volatiles by GC-MS and Variation in Total Volatile Content}

In the present study, we conducted a detailed volatile investigation of the metabolic response of cucumber leaves to N-sufficient and N-deficient treatments. The qualitative compounds were quantified and are listed in Table S1. A total of 24 volatile compounds were identified and quantified including 15 aldehydes, two ketones, two alkenes and five other volatile compounds. The amount of total volatiles accounted for 1914.71 to $4097.54 \mathrm{ng} / \mathrm{g}$ FW (fresh weight) in N-sufficient treatments and 411.73 to $5523.84 \mathrm{ng} / \mathrm{g}$ FW in N-deficient treatments. The lowest amount was detected at Day 7 during both treatments, and the highest content was detected at Days 6 and 5. At Days 1, 3, 6, and 7, the total amount of volatiles in $\mathrm{N}$-deficient nutrient solution was significantly lower than in plants which were cultivated in $\mathrm{N}$-sufficient nutrient solution. The total volatile content in the $\mathrm{N}$-deficient treatment clearly increased at Day 5 (Figure 1).

A wide variation in leaf volatile compounds was observed among the different nitrogen treatments (Table S1). During N-deficient treatments, all the volatile compounds displayed a significant difference compared to the N-sufficient treatments from Days 1 to 7 . Among the 24 volatile compounds, most showed lower level of volatile compounds in N-deficient treatments from Day 1 to Day 7 , with the exception of hexanal, $(E)$-2-hexenal, and $(E, E)$-2,4-hexadienal which belong to the $\mathrm{C} 6$ aldehydes and displayed higher levels at Days 5, 2 and 3, respectively (Figure S1). Interestingly, $\beta$-caryophyllene and $\alpha$-curcumene, which were detected in all N-sufficient leaves, were not found in any of the leaves from the $\mathrm{N}$-deficient treatments. 


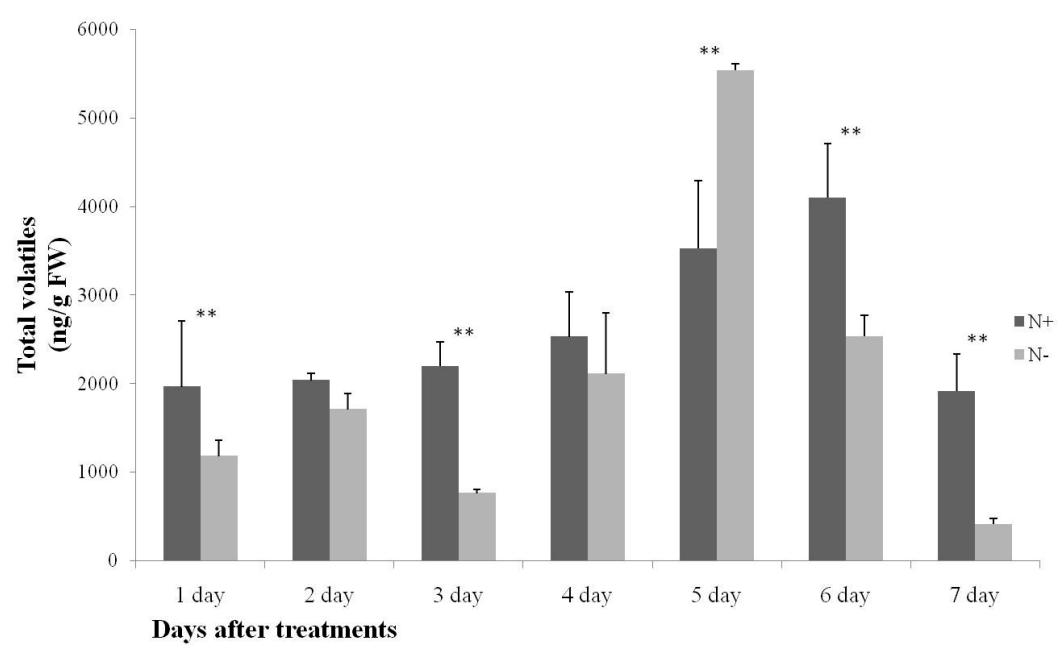

Figure 1. Changes of total volatile content in 9930 leaves during N-sufficient and N-deficient treatments. $\mathrm{N}+$ and $\mathrm{N}-$ mean nitrogen sufficient and nitrogen deficient treatments, respectively. Bars present means \pm standard deviations, data were analyzed by one-way ANOVA followed by the Duncan's multiple range tests to make comparisons within both treatments in same day. Asterisks indicate significant difference, ${ }^{* *} p<0.01$. Values are means of three replicates.

\subsection{Principal Component Analysis of Volatiles during N-Sufficient and N-Deficient Treatments}

Using Principal Component Analysis (PCA) to examine the data, PCA scores plots allow visual representation of any separation of cucumber leaf volatiles when comparing the $\mathrm{N}$-sufficient and $\mathrm{N}$-deficient treatments. In the present study, the complete data set was selected for PCA and was performed on an average content of all compounds from N-sufficient and N-deficient treatments. Principal Component 1 (PC1) and PC2 accounted for approximately $75.30 \%$ of the total variability. More specifically, PC1 accounted for $40.49 \%$, and PC2 was responsible for $34.81 \%$ (Figure 2).

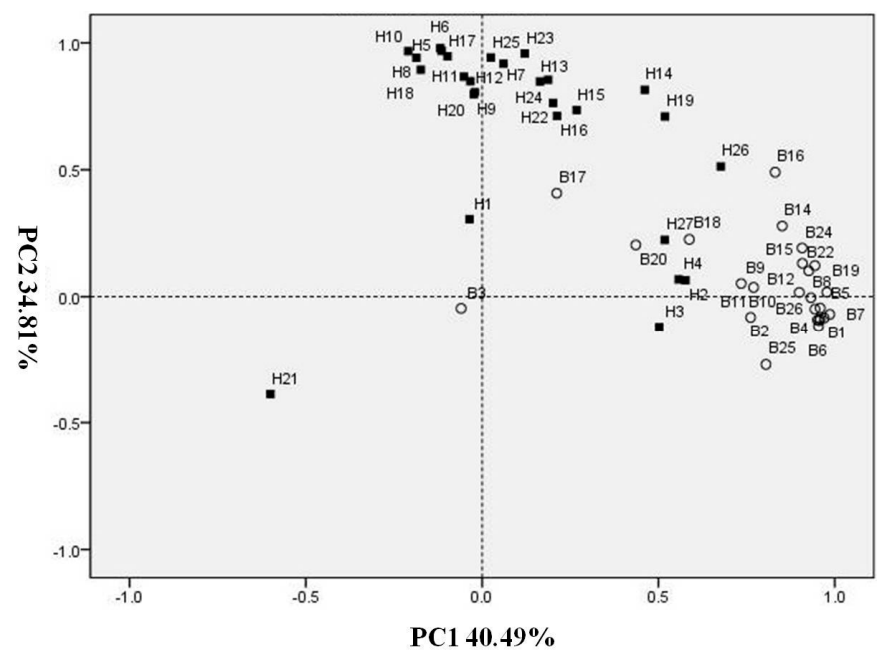

Figure 2. PCA of N-sufficient and deficient treatments based on the first 2 PCA results from volatile compounds. (H) Volatiles from N-sufficient treatments; (B) Volatiles from N-deficient treatments: 1, Hexanal; 2, (E)-2-Hexenal; 3, (E,E)-2,4-Hexadienal; 4, total C6 aldehydes; 5, (Z,Z)-3,6-nonadienal; 6, (Z)-6-Nonenal; 7, Nonanal; 8, (E,E)-2,6-Nonadienal; 9, (E,Z)-2,6-Nonadienal; 10, (E)-2-Nonenal; 11, Total C9 aldehydes; 12, Benzaldehyde; 13, $(E, E)-2,4$-Heptadienal; 14, Acetophenone; 15, (E,E)-3,5-Octadien-2-one; 16, 4-Heptenal; 17, Linalool; 18, Methyl salicylate; 19, $\beta$-Cyclocitral; 20, Undecanal; 21, $\beta$-Caryophyllene; 22, $\beta$-Ionone; 23, $\alpha$-Curcumene; 24, $\beta$-Ionone epoxide; 25, Tetradecanal; 26, (9E,12E,15E)-9,12,15-Octadecatrienal; 27, Total volatiles. 
Based on the loadings for this PCA, most volatiles of cucumber leaves grown in N-sufficient nutrition had higher concentration. When all volatiles of both $\mathrm{N}$-sufficient and $\mathrm{N}$-deficient treatments were combined, PCA using cucumber leaf composition parameters provided good separation (Figure 2). PCA on the profiles of volatile compounds resulted in a clustered pattern in which volatiles of the $\mathrm{N}$-deficient treatment were mainly clustered in the positive axis of PC1 and volatiles of $\mathrm{N}$-sufficient treatment were mainly clustered in the positive axis of PC2, with the exception of (E)-2-hexenal, $(E, E)$-2,4-hexadienal, total $\mathrm{C} 6$ aldehydes, and total volatiles, which displayed significant improvement in some points during $\mathrm{N}$-deficient treatment. These results indicated that nitrogen supply affected the production of most cucumber leaf volatiles.

\subsection{Changes of C6 and C9 Aldehyde Content during N-Deficient Treatment}

C6 and C9 aldehydes are known to contribute to the flavor and aroma of plants and resistance to pathogens and are powerful inducers of abiotic stress-related genes $[8,10,23]$. $\mathrm{C} 6$ aldehydes provide a more "green grass" flavor, while C9 aldehydes enhance the unique "cucumber-like" flavor [22]. In this study, C6 and C9 aldehydes constitute the majority of the cucumber leaf volatiles (Figure 3.); three C6 aldehydes were detected in cucumber leaves including hexanal, $(E)$-2-hexenal, and $(E, E)$-2,4-hexadienal. The proportion of these $\mathrm{C} 6$ compounds to total volatiles accounted for $77.96 \%$ to $91.11 \%$ in $\mathrm{N}$-sufficient treatments and $86.05 \%$ to $94.94 \%$ in $\mathrm{N}$-deficient treatments. Most of the relative composition of $\mathrm{C} 6$ aldehydes in $\mathrm{N}$-deficient treatments is higher than those that were $\mathrm{N}$-deficient, except on 7 day. The same trends of the $\mathrm{C} 6$ aldehyde ratio "low-high-low-high-low" pattern were observed during $\mathrm{N}$-sufficient and N-deficient treatments; this might be caused by difference of environment. Of the three $\mathrm{C} 6$ aldehydes, $(E)$-2-hexanal had the highest relative proportion, which accounted for $69.05 \%$ to $84.05 \%$.

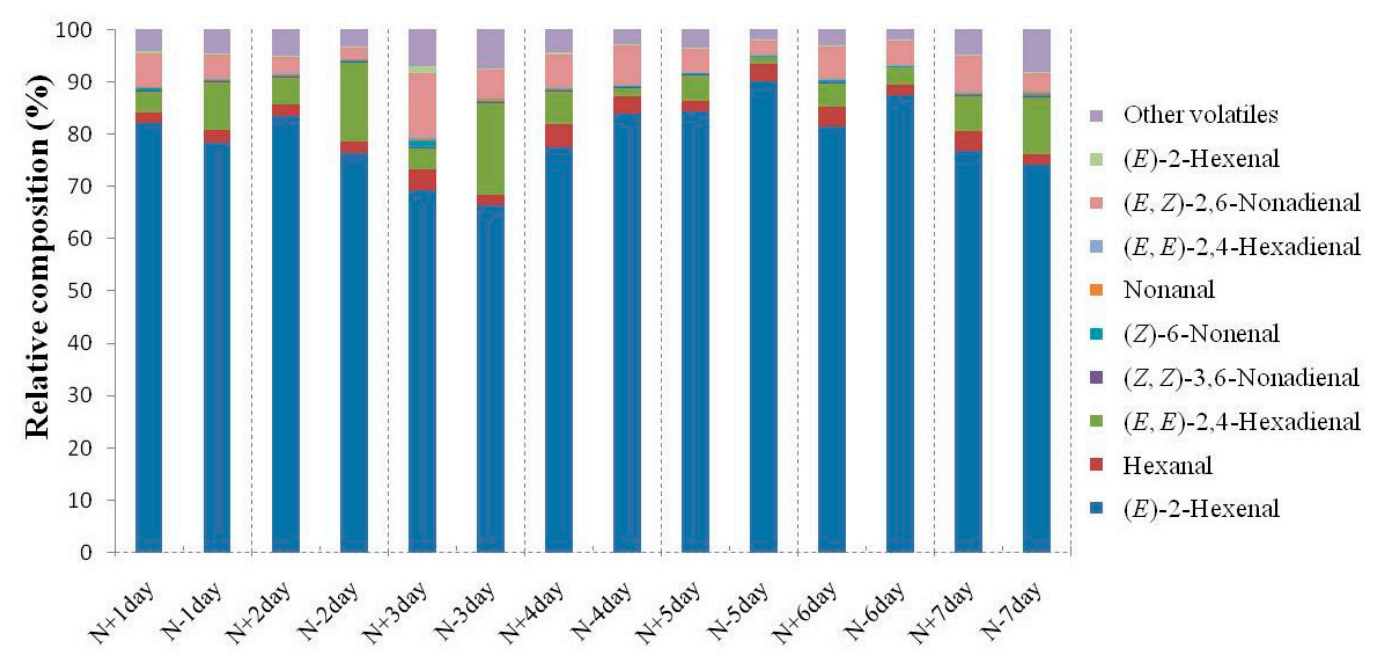

Days after treatments

Figure 3. Relative composition (\%) of volatiles from 9930 leaves of $\mathrm{N}$-sufficient and deficient treatments. $\mathrm{N}+$ and $\mathrm{N}-$ mean nitrogen sufficient and nitrogen deficient treatments, respectively. Values are means of three replicates.

Six C9 aldehydes were detected in cucumber leaves, including (E,Z)-2,6-nonadienal, ( $E, E)$-2,6-nonadienal, $(Z, Z)-3,6$-nonadienal, $(Z)-6$-nonenal, nonanal, and (E)-2-nonenal. The relative proportion of $C 9$ aldehydes accounted for $4.04 \%$ to $15.87 \%$ during $N$-sufficient treatments and for $2.84 \%$ to $8.42 \%$ during $\mathrm{N}$-deficient treatments. Among the six C9 aldehydes, (E,Z)-2,6-nonadienal had the highest proportion and accounted for $2.06 \%$ to $12.06 \%$ in all sample points. At four days after $\mathrm{N}$-deficient treatments, the $\mathrm{C} 9$ relative composition was higher than cultivated in $\mathrm{N}$-sufficient treatments, while the other six sampling points displayed lower values than controls. During 
$\mathrm{N}$-deficient treatments, the relative composition of $\mathrm{C} 6$ and $\mathrm{C} 9$ aldehydes were varied, the relative composition of $\mathrm{C} 6$ aldehydes was improved, and the amount of $\mathrm{C} 9$ aldehydes was reduced for most sample points.

Compared to $\mathrm{N}$-sufficient treatments, content of $(E, E)$-2,4-hexadienal displayed significant improvement at 2 and 3 day, hexanal and $(E)$-2-hexenal were also significantly improved at 5 day. (Figure 4). The amount of total C6 aldehydes ranged from 1696.69 to $3679.16 \mathrm{ng} / \mathrm{g}$ FW during the $\mathrm{N}$-sufficient treatments and from 358.87 to $5255.86 \mathrm{ng} / \mathrm{g}$ FW during N-deficient treatments. The amount of (E)-2-hexenal reached a maximum value of $4989.15 \mathrm{ng} / \mathrm{g}$ FW on Day 5. During N-deficient treatments, the amount of $(E)$-2-hexenal and hexanal displayed a decrease compared to N-sufficient treatments, with the exception of Day 5, on which a significant increase was displayed. The content of $(E, E)$-2,4-hexadienal displayed a different pattern during $\mathrm{N}$-deficient treatment, increasing from Day 1 to Day 3 and decreasing from Day 4 to Day 7.
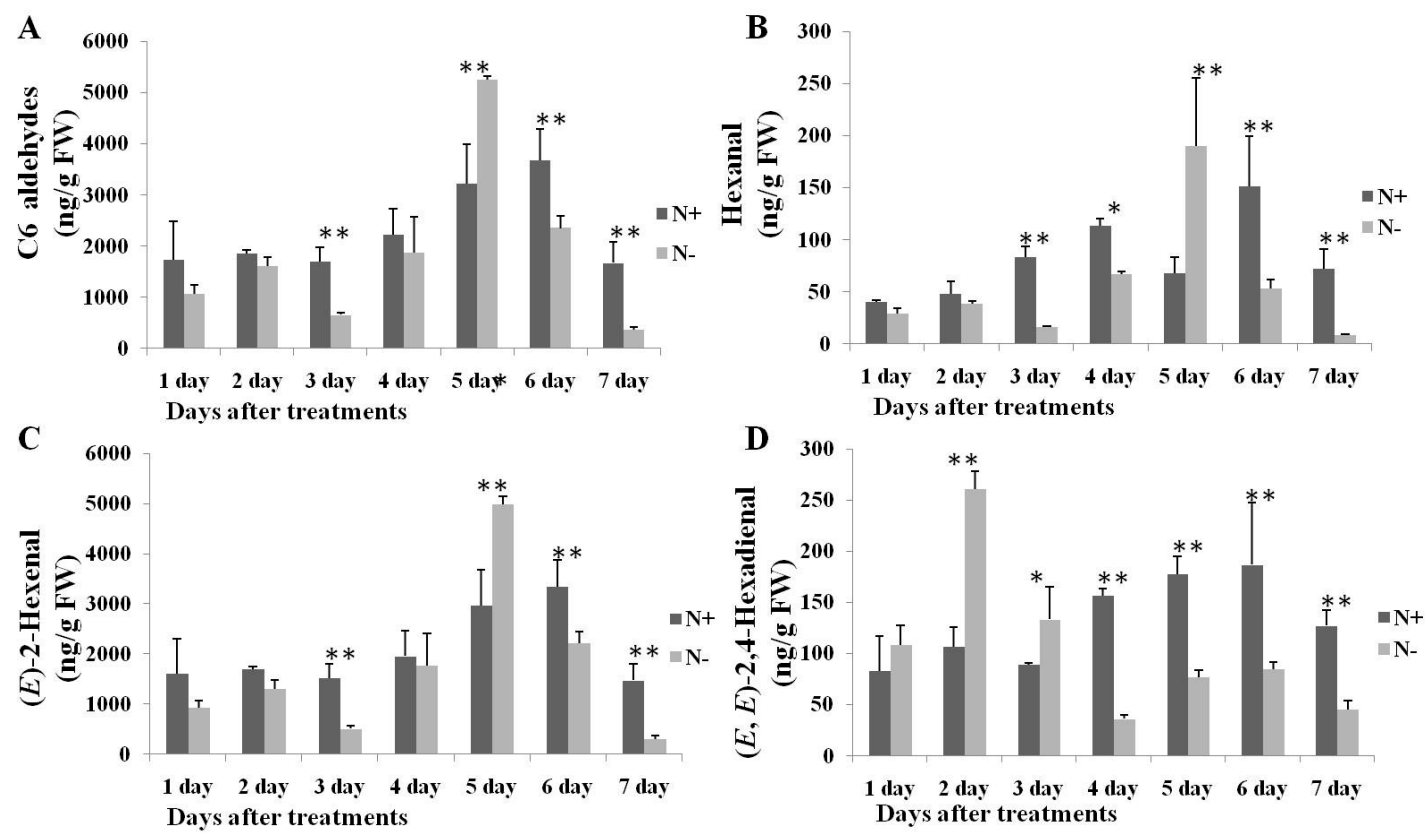

Figure 4. Changes in content of: C6 aldehydes (A); hexanal (B); (E)-2-hexenal (C); and $(E, E)-2,4-H e x a d i e n a l(D)$ in 9930 leaves during N-deficient treatments. Bars present means \pm standard deviations. ${ }^{*}$ and ${ }^{* *}$ mean significant difference from control at $p<0.05$ and $p<0.01$, respectively. $\mathrm{N}+$ and $\mathrm{N}-$ mean nitrogen sufficient and nitrogen deficient treatments, respectively. Values are means of three replicates.

During N-deficient treatments, the amount of $\mathrm{C} 9$ aldehydes also displayed significant changes compared to the control. As shown in Figure 5, the total amount of C9 aldehydes in N-deficient treatments was significantly reduced at Days 1,3,6, and 7. During the $\mathrm{N}$-sufficient and $\mathrm{N}$-deficient treatments, the highest amounts of C9 aldehydes were exhibited at Days 3 and 4 with 345.11 and $175.68 \mathrm{ng} / \mathrm{g} \mathrm{FW}$, respectively. (E,Z)-2,6-nonadienal was the main component of $C 9$ aldehydes, accounting for $70.13 \%$ to $88.74 \%$ of the total $C 9$ aldehydes. A higher ratio of $(E, Z)-2,6$-nonadienal between $\mathrm{N}$-sufficient and $\mathrm{N}$-deficient treatments was observed on Days 4 and 5, but no significant difference was observed. According to these results, we inferred that nitrogen acts as the key signal involved in the regulation of the short-chain volatile biosynthesis, not only for the amounts but also for the relative composition, and the amount of $\mathrm{C} 6$ aldehydes could be increased in response to N-deficiency. 


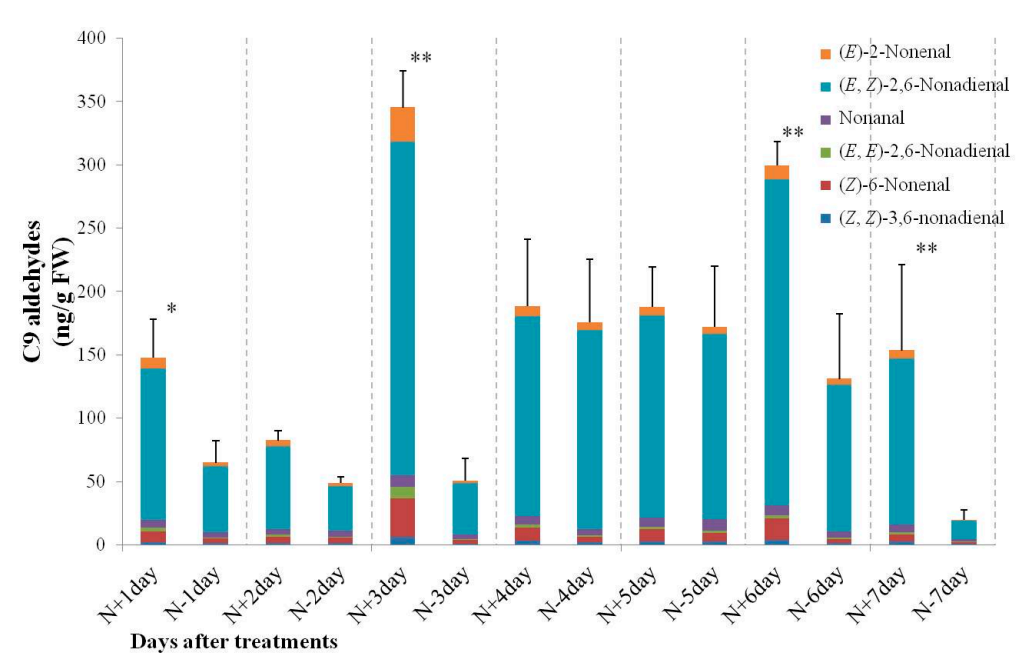

Figure 5. Changes in content of C9 aldehydes in 9930 leaves during $\mathrm{N}$-sufficient and deficient treatments. Bars present means \pm standard deviations. ${ }^{*}$ and ${ }^{* *}$ mean significant difference from control at $p<0.05$ and $p<0.01$, respectively. $\mathrm{N}+$ and $\mathrm{N}-$ mean nitrogen sufficient and nitrogen deficient treatments, respectively. Values are means of three replicates.

\subsection{Relationship between $C 6$ and C9 Aldehydes and Gene Expressions in the 9930 Line}

In order to explore potential correlations between aldehydes, the folds of CsLOXs and CsHPLs gene expression patterns were used for HCA analysis, and $\mathrm{N}$-deficient treatments were compared to N-sufficient treatments. HCA data resulted in two distinct sample clusters (Figure 6). Cluster I included six CsLOXs (CsLOX2, 9, 17, 19, 20, and 22) that displayed higher up-regulated expression patterns during $\mathrm{N}$-deficient treatments. Cluster II included all nine aldehydes, six CsLOXs, and two CsHPLs. The expression of CsHPL1 was significantly increased at $5 \mathrm{~d}$, meanwhile the amount of (E)-2-hexenal and hexanal were also significantly up-regulated. This result indicated that CsLOX2, 9, $17,19,20$, and 22 were responsive to $\mathrm{N}$-deficiency and had higher up-regulated expression patterns during short-term $\mathrm{N}$-deficient treatments, thus the increase in $\mathrm{C} 6$ aldehydes might be induced by the up-regulation of CSHPL1.

\subsection{Changes of C6 and C9 Volatile Content during N-Deficient Treatment in Six Other Inbred Cucumber Lines}

In order to further study the response of the $\mathrm{C} 6$ and $\mathrm{C} 9$ volatile metabolism to $\mathrm{N}$-deficient treatments, six other inbred cucumber lines were cultivated in N-deficient nutrition solution and sampled at Day 5 for detecting leaf volatiles. Seven short-chain leaf volatiles (hexanal, $(E, E)$-2,4-hexadienal, $(E)$-2-hexenal, nonanal, (Z)-6-nonenal, (E,Z)-2,6-nonadienal, and (E,Z)-2,6-nonadienol) were detected in cucumber leaves, as shown in Figure 7 and Table S3, compared to N-sufficient treatments, the total amount of short-chain volatiles of all six lines was significantly improved in the $\mathrm{N}$-deficient treatments, up-regulation of 0.87 - to 1.96 -fold was displayed, and the amount of $\mathrm{C} 6$ volatiles of all the six lines and C9 volatiles of five lines increased. Of the six inbred lines, (E)-2-hexenal was still the main component, displaying as much as $75.38 \%$ in all treatments. (E,Z)-2,6-nonadienol was the smallest component, displaying between only $0.02 \%$ and $0.73 \%$ in all treatments. During $\mathrm{N}$-deficient treatments, changes in volatile components were observed among six inbred lines: 32 volatiles were up-regulated and 10 volatiles were down-regulated at 5 day. All of the (E)-2-hexenal amounts, which had green-like flavor, as well as (E,Z)-2,6-nonadienal, which had cucumber-like flavor, were improved compared to $\mathrm{N}$-sufficient treatments. The other five volatiles displayed down-regulation in some lines. Among the six inbred lines, different responses to N-deficiency were also observed. For A37 and A91, the amount of all seven volatiles increased. For A74, only one volatile decreased, but for A23, the content of (E)-2-hexenal, (E,Z)-2,6-nonadienol, and (E,Z)-2,6-nonadienal displayed up-regulation, whereas the amount of the other four volatiles decreased. 


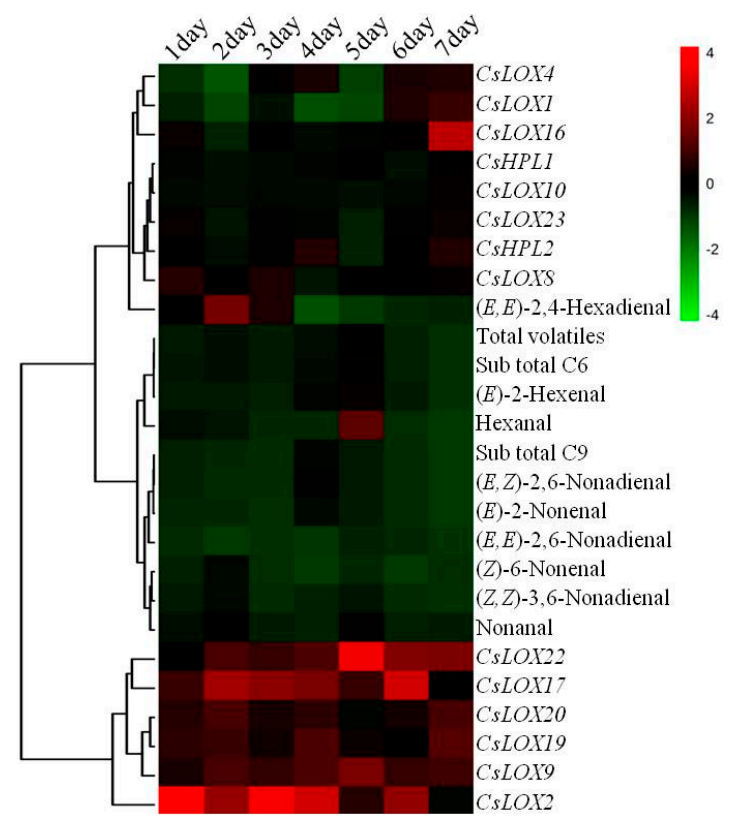

Figure 6. Hierarchical cluster dendrogram and heat map of volatiles content and gene expression folds during $\mathrm{N}$-sufficient and deficient treatments in 9930 leaves. Leaves were sampled at 1, 2, 3, 4, 5, 6 and 7 day after $\mathrm{N}$-deficient treatments. The heat map has been generated based on the fold-change values of volatile contents, CsLOXs and CsHPLs expression level in the treated samples when compared with its unstressed control samples by MetaboAnalyst 3.0 software. The color scale for fold-change values is shown on the right. Values of gene expression folds are means of three biological replicates with three technical replicates. Values of short-chain volatiles are means of three biological replicates.

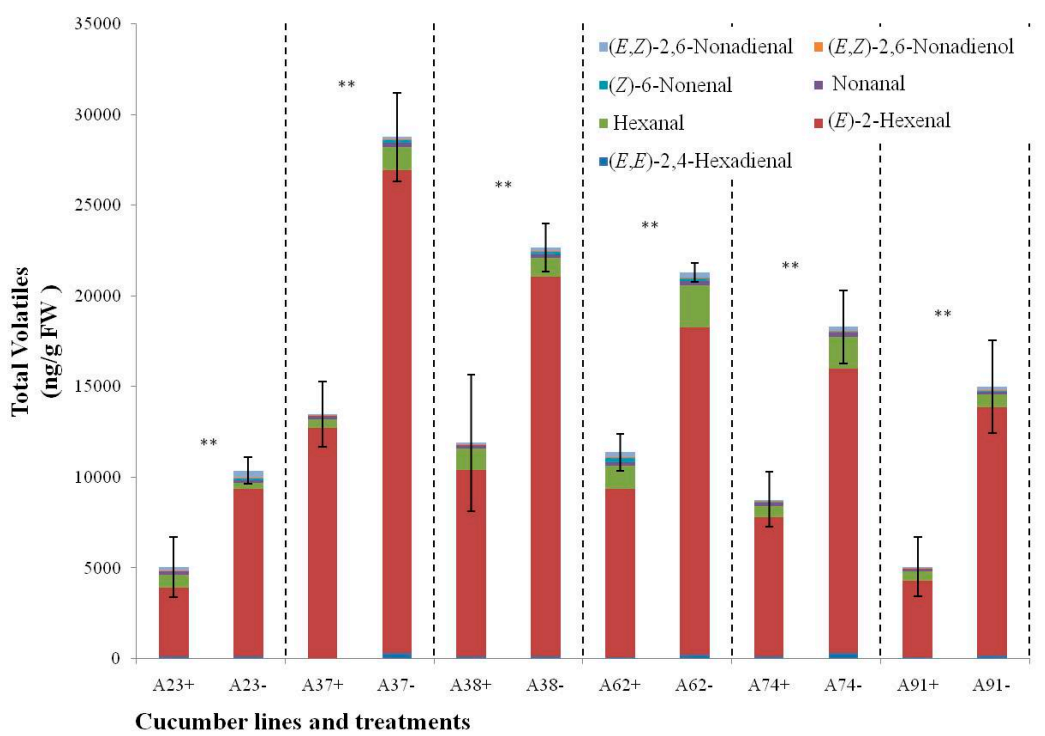

Figure 7. Changes in content of short-chain volatiles in six inbred cucumber leaves at 5 day after $\mathrm{N}$-deficient treatments. Bars present means \pm standard deviations. + and - mean $\mathrm{N}$-sufficient and deficient treatments, respectively. ${ }^{* *}$ mean significant difference from control at $p<0.01$. Values are means of three biological replicates.

Combined with the changes of 9930 and the volatile content of six other inbred lines, we suggest that the amount of short-chain leaf volatiles in cucumber seedlings could be significantly improved in response to nitrogen deficiency. A previous study showed that reactive short-chain leaf volatiles 
(RSLVs) such as (E)-2-hexenal can act as signal chemicals that strongly induce the gene expression of abiotic-related transcription factors. (E)-2-hexenal could up-regulate genes that respond to salt, oxidation, osmotic drought, cold, and wounding [21]. In the present study, RSLVs might involve in the induction of the expression of down-stream transcription factors during nitrogen starvation.

\subsection{The Expression Patterns of Cucumber LOX Genes in Response to N-Deficient Treatments in Six Inbred Cucumber Lines}

LOX proteins and their metabolites have been demonstrated to involve in production of short-chain volatile compounds [11] and plant defense responses during various stresses [24,25]. To study the effect of nitrogen deficiency on the biosynthesis of volatiles in cucumber seedlings, LOX gene expression profiles in cucumber leaves were analyzed by qPCR.

Of the 12 LOX genes, six CsLOXs (CsLOX1, 2, 4, 8, 9, and 10) were predicted to be type-1 LOXs, which are involved in the biosynthesis of C9 aldehydes. The other six LOXs (CsLOX16, 17, 19, 20, 22, and 23) were predicted to be type-2, which are involved in the biosynthesis of C6 aldehydes [23]. In our study, compared to N-sufficient treatments in six inbred cucumber lines, 157 of 276 sample points of CsLOXs expressed higher levels during N-deficient treatments (Table S2), including 68 type-1 CsLOXs and 89 type-2 CsLOXs sample points. Two types of CsLOXs displayed a clear difference in response to $\mathrm{N}$-deficiency treatments, and type-2 CsLOXs had more up-regulated expression patterns. Of the six inbred lines, different expression patterns of CsLOXs were observed during $\mathrm{N}$-deficient treatments. For A23, CsLOX9 had higher up-regulated patterns; for A74 and A91, CsLOX19, 20, and 22 displayed higher up-regulation. For A38, CsLOX10 displayed up-regulation (Table S2).

HCA was employed to explore potential correlations among 12 CsLOXs, and, to stratify samples based on trait, Ward's linkages in Euclidian distance were used. HCA data resulted in two distinct sample clusters (Figure 8). Cluster I included 6 CsLOXs (CsLOX8, 10, 19, 20, 22, and 23) which displayed more up-regulated expression patterns during N-deficient treatments. The six other CsLOXs belonged to Cluster II and displayed more down-regulated patterns.

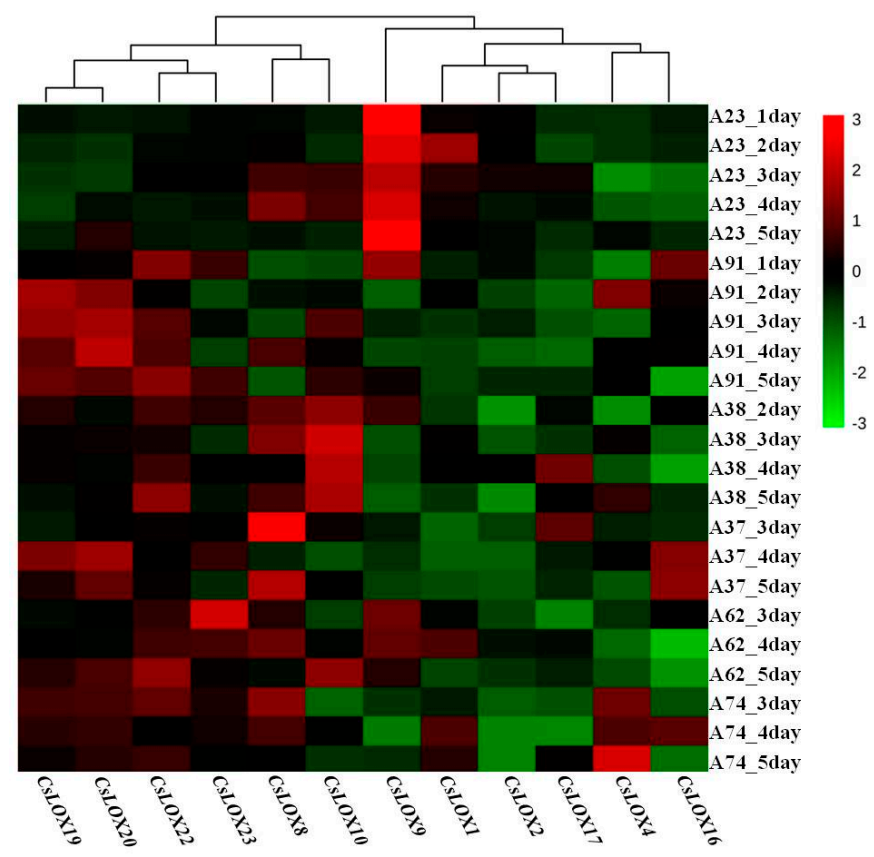

Figure 8. Hierarchical cluster dendrogram and heatmap of CsLOXs expression folds in six inbred cucumber leaves during $\mathrm{N}$-deficient treatments. The heat map has been generated based on the fold-change values of CsLOXs expression level in the treated samples when compared with its unstressed control samples. The color scale for fold-change values is shown the right. Values are means of three biological replicates with three technical replicates. 
A previous study showed that LOXs were involved in the response to abiotic stress and plant growth regulators [11]. Our results from the expression of CsLOXs demonstrated that type- 1 and type-2 CsLOXs had different response patterns to short term N-deficiency. In most sample points during $\mathrm{N}$-deficient treatments, type-1 CsLOXs were down-regulated and type-2 CsLOXs were up-regulated. Combined with the expression patterns of the 9930 line, we suggest that CsLOX19, CsLOX20, and CsLOX22 which belong to type-2 CsLOXs might be involved in the response to $\mathrm{N}$-deficiency and the improvement of $\mathrm{C} 6$ aldehydes content might be partly caused by the up-regulation of type- 2 CsLOXs in cucumber leaves.

\subsection{The Expression Patterns of CsHPLs during N-Deficient Treatments in Six Inbred Cucumber Lines}

In the LOX-HPL pathway, HPL is one of the key enzymes that catalyzes 13-HPOD/HPOT or 9-HPOD/HPOT to produce short-chain volatiles. In cucumber, three HPL genes were divided into three types by their specificities of substrate: 13-HPL which specifically catalyzes 13-HPOD/HPOT to produce $\mathrm{C} 6$ and $\mathrm{C} 12$ volatiles, 9-HPL which catalyzes 9-hydroperoxides to form $\mathrm{C} 9$ aldehydes, and 9/13-HPL, which can use both 9- and 13-hydroperoxides as substrates to produce C6 and C9 aldehydes [22,25]. To study the changes in the expression patterns of CsHPLs during N-deficient treatments, HPLs gene expression profiles in cucumber leaves were analyzed by qPCR.

In our study, only CSHPL1 (13-HPL) and CsHPL2 (9/13-HPL) were expressed in cucumber leaves. For the six inbred lines, as shown in Figure 9, CsHPL1 had a higher expression level than CsHPL2 and most sample points of CsHPL1 were significantly up-regulated during N-deficient treatments; no significantly reduced points were observed. For CsHPL2, 13 out of 23 sample points were significantly up-regulated during $\mathrm{N}$-deficient treatments, and only one point was down-regulated significantly. For A91, all the sample points of CsHPL1 and CsHPL2 were significantly up-regulated compared to $\mathrm{N}$-deficient treatments.
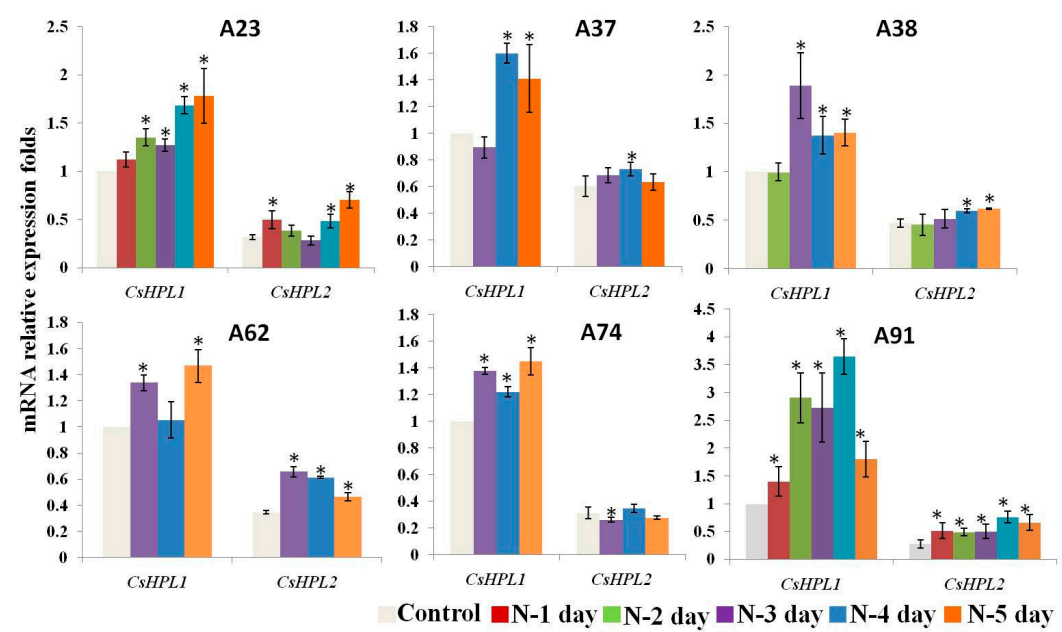

Figure 9. Expression profiles of HPL genes in six inbred cucumber leaves during N-deficient treatments. Leaves were sampled at 1, 2, 3, 4 and $5 \mathrm{~d}$ after $\mathrm{N}$-deficient treatments. The map has been generated based on the fold-change values of CsHPLs in the treated samples when compared with its unstressed control samples. The CsHPL1 expression level of control was considered as one-fold. Bars present means \pm standard deviations. * means significant difference from control at $p<0.05$. Values are means of three biological replicates with three technical replicates.

Our results show that content of short-chain volatiles was significantly improved at Day 5; meanwhile, the expression pattern of CSHPL1 was also significantly improved in all seven inbred lines. A previous study showed that $C 9$ aldehydes are the major volatiles in cucumber fruit [26]. The loss of 9-HPL enzyme activity and the fact that 9/13-HPL enzyme preferentially exhibits 13-HPL enzyme activity [22] in cucumber leaves might be the main reasons for the relative composition difference of C6 
and C9 aldehydes between cucumber fruit and leaves. According to these results, we suggest that the significant improvement of short-chain volatiles in cucumber was partly caused by the up-regulated expression of CsHPL1 during N-deficient treatments.

\section{Material and Methods}

\subsection{Plant Materials and N-Deficient Treatments}

Cucumber (Cucumis sativus) cv. Lines 9930 were grown on 15 March 2015 and harvested on 23 April. The other six inbred lines (A23, A37, A38, A62, A74 and A91, cultivars of northern China) were grown 25 October and harvested on 10 December. Seeds were surface sterilized with $5 \%(w / v)$ $\mathrm{NaClO}$ for $15 \mathrm{~min}$ and germinated on wet filter paper in the dark. Cucumber plants were then moved into a sponge floating and cultivated in a hydroponic system containing modified Hoagland solution (11 $\mathrm{mM} \mathrm{NO}_{3}{ }^{-}, 1 \mathrm{mM} \mathrm{NH}_{4}{ }^{+}, \mathrm{pH}$ 6.0) in a green house. The solutions were renewed every three or four days. After three true leaves reached, cucumber seedlings were moved into an $\mathrm{N}$-free nutrient solution as $\mathrm{N}$-deficiency treatment $\left(0 \mathrm{mM} \mathrm{NO}_{3}{ }^{-}, 0 \mathrm{mM} \mathrm{NH}_{4}{ }^{+} ; \mathrm{Ca}\left(\mathrm{NO}_{3}\right)_{2}\right.$ was replaced with $\mathrm{CaCl}_{2}$; other ions were same as $\mathrm{N}$-sufficient conditions except for chlorid ion (Table S4)), and plants cultivated in N-sufficient conditions as controls. The second fully expanded apical leaves were harvested and immediately frozen in liquid nitrogen and stored at $-80{ }^{\circ} \mathrm{C}$ until analysis. Plant samples were taken with three biological replicates.

\subsection{HS-SPME Extraction}

Cucumber leaves were fully grinded in liquid nitrogen and then two hundred microgram sample was put into a glass vial, $0.4 \mathrm{~mL}$ of saturated sodium chloride solution which contained $50 \mathrm{ng}$ of 2-Heptanonel as an internal standard were added to terminate enzymatic reaction and improve the volatiles into the headspace. After micro-extraction, volatiles were transferred into a head vial with crimp cap, and then fifty/thirty micromoles of CAR/DVB/PMDS fiber (Supleco, Bellefonte, PA, USA) were used for the next analysis. Samples were continuous agitated at $40{ }^{\circ} \mathrm{C}$ for $30 \mathrm{~min}(600 \mathrm{rpm})$, volatile compounds were also extracted under the same conditions.

\subsection{GC-MS Analysis}

Samples were analyzed by 7890A GC gas chromatograph combined with a 5975C mass spectrometer (Agilent Technologies, Santa Clara, CA, USA) on a DB5-MS capillary column ( $30 \mathrm{~m} \times 0.25 \mathrm{~mm} \times 0.25 \mu \mathrm{m}$ with $5 \%$ Phenyl methyl siloxane, J \& W Scientific, Folsom, CA, USA). GC condition was: helium was the carrier gas and flow rate through the column was $1.5 \mathrm{~mL}$ per min. The oven temperature programmed at $40^{\circ} \mathrm{C}$ for $1.5 \mathrm{~min}$, then ramped up to $150{ }^{\circ} \mathrm{C}$ at $5{ }^{\circ} \mathrm{C}$ per minute, after holding there for $13 \mathrm{~min}$, ramped up to $230^{\circ} \mathrm{C}$ at $15^{\circ} \mathrm{C}$ per minute, and held for $15 \mathrm{~min}$. Mass spectra were obtained at $70 \mathrm{eV}$.

\section{4. $R N A$ Isolation and $q P C R$}

Total RNA was isolated from cucumber leaves of seedlings using the Trizol reagent (Invitrogen, Carlsbad, CA, USA) according to the manufacturer's instructions. Concentration and purity of RNA were determined with a NanoDrop ND-1000 photospectrometer. cDNA was synthesized with $5 \mu \mathrm{g}$ of total RNA using the M-MLV reverse transcriptase (Promega, Madison, WI, USA). The specific primers of 23 CsLOX genes were synthesized by previous study [11]. Three cucumber HPL gene sequences were downloaded from Cucumber Genome DataBase [27], the specific primers were designed using DNAMAN 6.0 (Lynnon Biosoft, San Ramon, CA, USA) for RT-PCR and qPCR (CsHPL1-F: TACCTTCATCTATTTCCCCC, CsHPL1-R: GAAGAACTTATCGGGTCCTT; CsHPL2-F: CCCGAATTACCAAATACAAC, CsHPL2-R: TAAGTTCCGTCGAGAATGTT; CsHPL3-F: TCATCTTCTTCAGAACACCC, CsHPL3-R: GATCGGAAGAAGGTTTCTCT). The amplified PCR products were quantified by an Applied BioSystems 7500 Real Time PCR System (Bio-Rad Laboratories, 
Hercules, CA, USA), with a SYBR Premix Ex Taq kit (Takara, Tianjin, China). All qPCR analyses were performed in three biological replicates with three technical replicates and the results were generated using 7500 software (Bio-Rad Laboratories, Hercules, CA, USA). For relative quantification, CsACT (Csa2M301530) (for the quantitation of gene expression during N-deficient treatments) gene was detected as an internal reference, and the $2^{-\Delta \Delta C t}$ method was used. PCR conditions were as follows: 5 min initial denaturation at $95^{\circ} \mathrm{C}$ followed by 30 cycles of denaturation at $94{ }^{\circ} \mathrm{C}$ for $30 \mathrm{~s}$, annealing at $50{ }^{\circ} \mathrm{C}$ for $30 \mathrm{~s}$ and an extension at $72{ }^{\circ} \mathrm{C}$ for $2 \mathrm{~min}$. qPCR analysis was performed according to Zhao et al. [3].

\subsection{Statistical Analysis}

GC-MS analysis was performed according to Azam et al. [28], and the 2-Heptanone was used as internal standard for calculating content of volatiles. All data were expressed as the means \pm SD of three replicates and used for multivariate analysis. Statistical analysis was performed using ANOVA within SPSS 22.0 (IBM, Armonk, NY, USA). Significant differences between leaves at different nitrogen treatments were confirmed using the Duncan's multiple range test. Principal component analysis (PCA) was used to detect clustering and to investigate possible relationships between different nitrogen treatments and volatile compounds. Heat map generation and hierarchical cluster analysis (HCA) were carried out using MetaboAnalyst 3.0 software [29].

\section{Conclusions}

Volatiles of cucumber leaves in 9930 and six other inbred lines were identified, and the expression patterns of 12 CsLOXs and two CsHPLs during nitrogen-deficient treatments were also detected by $\mathrm{qPCR}$. The results indicate that the amount of short-chain volatiles was significantly improved during $\mathrm{N}$-deficient treatments at 5 day, while other volatiles had no significant improvement at all sampling points. CsLOXs and CsHPLs displayed differential expression patterns in response to nitrogen deficiency. According to the results, we suggest that CsLOX19, CsLOX20, and CsLOX22, three type-2 LOXs predicted to be 13-LOX, and CsHPL1 might be involved in response to N-deficiency and the increase of short-chain volatiles in cucumber leaves. The present work provides a valuable contribution toward the relationship between nitrogen and leaf volatiles and also enriches the databank of leaf volatiles. In addition, it can provide a more complete understanding of the LOX-HPL gene regulation patterns during nitrogen-deficient treatments.

Supplementary Materials: Supplementary materials can be found at www.mdpi.com/1422-0067/17/11/1713/s1.

Acknowledgments: This work was supported by the National Natural Science Foundation of China (No. 31672203, and No. 31471920) and Collaborative innovation center of protected vegetable suround Bohai gulf region. We sincerely thank Guo-dong Wang, Institute of Genetics and Developmental Biology, Chinese Academy of Sciences for detecting volatiles.

Author Contributions: Jie Deng and Hong-Jun Yu designed and performed the experiments, analyzed the data, and co-wrote the manuscript. Yun-Yun Li, Xiao-Meng Zhang, and Peng Liu helped with collecting materials, extracting total RNA, and performing RT-PCR and qPCR. Qiang Li and Wei-Jie Jiang supervised the data collection and revised the manuscript. All authors have read and approved the final manuscript.

Conflicts of Interest: The authors declare no conflict of interest.

\section{References}

1. Palma-Harris, C.; McFeeters, R.F.; Fleming, H.P. Solid-phase microextraction (SPME) technique for measurement of generation of fresh cucumber flavor compounds. J. Agric. Food Chem. 2001, 49, 4203-4207. [CrossRef] [PubMed]

2. Liu, J.G.; Diamond, J. China's environment in a globalizing world. Nature 2005, 435, 1179-1186. [CrossRef] [PubMed] 
3. Zhao, W.C.; Yang, X.Y.; Yu, H.J.; Jiang, W.J.; Sun, N.; Liu, X.R.; Liu, X.L.; Zhang, X.M.; Wang, Y.; Gu, X.F. RNA-Seq-Based Transcriptome Profiling of Early Nitrogen Deficiency Response in Cucumber Seedlings Provides New Insight into the Putative Nitrogen Regulatory Network. Plant Cell Physiol. 2015, 56, 455-467. [CrossRef] [PubMed]

4. Wang, Y.T.; Huang, S.W.; Liu, R.L.; Jin, J.Y. Effects of nitrogen application on flavor compounds of cherry tomato fruits. J. Plant Nutr. Soil Sci. 2007, 170, 461-468. [CrossRef]

5. Hoffland, E.; van Beusichem, M.L.; Jeger, M.J. Nitrogen availability and susceptibility of tomato leaves to Botrytis cinerea. Plant Soil 1999, 210, 263-272. [CrossRef]

6. Zafar, Z.U.; Athar, H.U.R.; Ashraf, M. Responses of Two Cotton (Gossypium hirsutum L.) Cultivars Differing in Resistance to Leaf Curl Virus Disease to Nitrogen Nutrition. Pak. J. Bot. 2010, 42, 2085-2094.

7. Zhang, X.; Yu, H.J.; Zhang, X.M.; Yang, X.Y.; Zhao, W.C.; Li, Q.; Jiang, W.J. Effect of nitrogen deficiency on ascorbic acid biosynthesis and recycling pathway in cucumber seedlings. Plant Physiol. Biochem. 2016, 108, 222-230. [CrossRef] [PubMed]

8. Chen, S.; Zhang, R.; Hao, L.; Chen, W.; Cheng, S. Profiling of volatile compounds and associated gene expression and enzyme activity during fruit development in two cucumber cultivars. PLOS ONE 2015. [CrossRef] [PubMed]

9. Siangdung, W.; Fukushige, H.; Hildebrand, D. Hydroperoxidelyase and leaf aldehyde formation can be greatly increased in leaves. Adv. Res. Plant Lipids 2003. [CrossRef]

10. Scala, A.; Mirabella, R.; Mugo, C.; Matsui, K.; Haring, M.A.; Schuurink, R.C. E-2-hexenal promotes susceptibility to Pseudomonas syringae by activating jasmonic acid pathways in Arabidopsis. Front. Plant Sci. 2013. [CrossRef] [PubMed]

11. Yang, X.Y.; Jiang, W.J.; Yu, H.J. The Expression Profiling of the Lipoxygenase (LOX) Family Genes during Fruit Development, Abiotic Stress and Hormonal Treatments in Cucumber (Cucumis sativus L.). Int. J. Mol. Sci. 2012, 13, 2481-2500. [CrossRef] [PubMed]

12. Forss, D.; Dunstone, E.; Ramshaw, E.; Stark, W. The flavor of cucumbers. J. Food Sci. 1962, $27,90-93$. [CrossRef]

13. Engelberth, J.; Alborn, H.T.; Schmelz, E.A.; Tumlinson, J.H. Airborne signals prime plants against insect herbivore attack. Proc. Natl. Acad. Sci. USA 2004, 101, 1781-1785. [CrossRef] [PubMed]

14. Ruther, J.; Furstenau, B. Emission of herbivore-induced volatiles in absence of a herbivore-Response of Zea mays to green leaf volatiles and terpenoids. Z. Naturforsch. C. 2005, 60, 743-756. [CrossRef] [PubMed]

15. Ruther, J.; Kleier, S. Plant-plant signaling: Ethylene synergizes volatile emission in Zea mays induced by exposure to (Z)-3-Hexen-1-ol. J. Chem. Ecol. 2005, 31, 2217-2222. [CrossRef] [PubMed]

16. Croft, K.; Juttner, F.; Slusarenko, A.J. Volatile Products of the Lipoxygenase Pathway Evolved from Phaseolus vulgaris (L.) Leaves Inoculated with Pseudomonas syringae pv. phaseolicola. Plant Physiol. 1993, 101, 13-24. [PubMed]

17. Shiojiri, K.; Ozawa, R.; Matsui, K.; Kishimoto, K.; Kugimiya, S.; Takabayashi, J. Role of the lipoxygenase/lyase pathway of host-food plants in the host searching behavior of two parasitoid species, Cotesia glomerata and Cotesia plutellae. J. Chem. Ecol. 1996, 32, 969-979. [CrossRef] [PubMed]

18. Heiden, A.C.; Kobel, K.; Langebartels, C.; Schuh-Thomas, G.; Wildt, J. Emissions of oxygenated volatile organic compounds from plants-Part I: Emissions from lipoxygenase activity. J. Atmos. Chem. 2003, 45, 143-172. [CrossRef]

19. Kishimoto, K.; Matsui, K.; Ozawa, R.; Takabayashi, J. Volatile C6-aldehydes and Allo-ocimene activate defense genes and induce resistance against Botrytis cinerea in Arabidopsis thaliana. Plant Cell Physiol. 2005, 46, 1093-1102. [CrossRef] [PubMed]

20. Noordermeer, M.A.; Veldink, G.A.; Vliegenthart, J.F. Fatty acid hydroperoxidelyase: A plant cytochrome p450 enzyme involved in wound healing and pest resistance. Chembiochem 2001, 2, 494-504. [CrossRef]

21. Yamauchi, Y.; Kunishima, M.; Mizutani, M.; Sugimoto, Y. Reactive short-chain leaf volatiles act as powerful inducers of abiotic stress-related gene expression. Sci. Rep. 2015. [CrossRef] [PubMed]

22. Wan, X.H.; Chen, S.X.; Wang, C.Y.; Zhang, R.R.; Cheng, S.Q. Isolation, expression, and characterization of a hydroperoxidelyase gene from cucumber. Int. J. Mol. Sci. 2013, 14, 22082-22101. [CrossRef] [PubMed]

23. Liavonchanka, A.; Feussner, N. Lipoxygenases: Occurrence, functions and catalysis. J. Plant Physiol. 2006, 163, 348-357. [CrossRef] [PubMed] 
24. Porta, H.; Rocha-Sosa, M. Plant lipoxygenases. Physiological and molecular features. Plant Physiol. 2002, 130, 15-21. [CrossRef] [PubMed]

25. Matsui, K.; Ujita, C.; Fujimoto, S.; Wilkinson, J.; Hiatt, B.; Knauf, V.; Kajiwara, T.; Feussner, I. Fatty acid 9- and 13-hydroperoxide lyases from cucumber. FEBS Lett. 2000, 481, 183-188. [CrossRef]

26. Buescher, R.H.; Buescher, R.W. Production and stability of $(E, Z)-2,6$-nonadienal, the major flavor volatile of cucumbers. J. Food. Sci. 2001, 66, 357-361. [CrossRef]

27. Liu, S.Q.; Liu, X.H.; Jiang, L.W. Genome-wide identification, phylogeny and expression analysis of the lipoxygenase gene family in cucumber. Genet. Mol. Res. 2011, 10, 2613-2636. [CrossRef] [PubMed]

28. Azam, M.; Jiang, Q.; Zhang, B.; Xu, C.J.; Chen, K.S. Citrus Leaf Volatiles as Affected by Developmental Stage and Genetic Type. Int. J. Mol. Sci. 2013, 14, 17744-17766. [CrossRef] [PubMed]

29. Xia, J.; Sinelnikov, I.; Han, B.; Wishart, D.S. MetaboAnalyst 3.0-Making metabolomics more meaningful. Nucleic Acids Res. 2015, 43, 251-257. [CrossRef] [PubMed]

(C) 2016 by the authors; licensee MDPI, Basel, Switzerland. This article is an open access article distributed under the terms and conditions of the Creative Commons Attribution (CC-BY) license (http://creativecommons.org/licenses/by/4.0/). 